\title{
Escala para medir tecnodependencia en el ámbito personal, familiar, social y laboral en población mexicana
}

\section{Technodependence in personal, familiar, social and labor sphere in Mexican population measuring scale} \section{(D) PsicoqENTE}

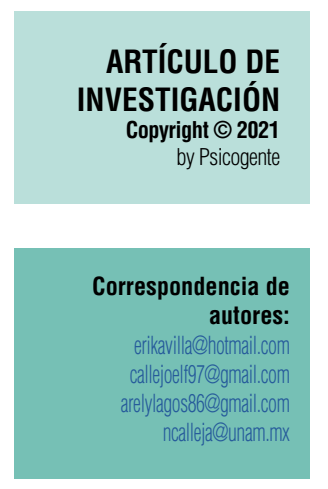

Recibido: 02-10-20 Aceptado: $21-09-21$ Publicado: $01-10-21$

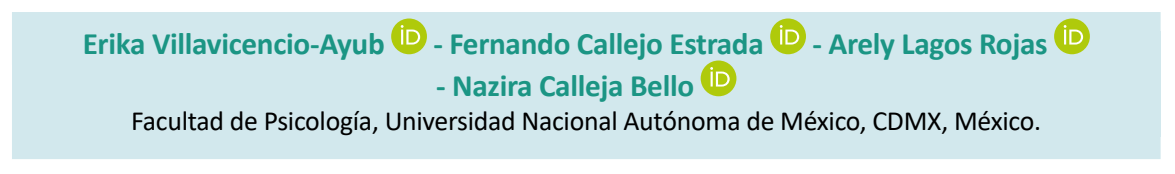

\section{Resumen}

Introducción: El uso de la tecnología se ha posicionado como una herramienta indispensable en la actualidad en ámbitos laborales, escolares y hasta en las interacciones sociales. Con ello el involucramiento con las tecnológicas permiten desempeñarnos con mayor productividad y eficiencia; sin embargo, un uso desadaptativo puede propiciar diversas afecciones que inciden en la salud y relaciones de los usuarios.

Objetivo: Se diseñó una escala para medir tecnodependencia en las personas, conformada por cinco factores que fueron nombrados como Phubbing, Generación Muda, Uso de la Tecnología al Conducir Uso compulsivo del Celular y la Vida en Redes Sociales.

Método: Estudio cuantitativo, transversal, con diseño no experimental y muestreo no probabilístico, que contó con 1026 participantes, de los cuales el 63 \% correspondía a hombres. Se utilizó la Escala de Tecnodependencia elaborada por los autores para el presente estudio, conformada por un total de 15 reactivos divididos en cinco factores (Uso del celular al conducir, Vida en redes sociales, Generación Muda, Uso compulsivo del celular y Phubbing), además de las variables sociodemográficas, con un índice de confiabilidad de 0,83.

Resultados: Se encontró correlación estadísticamente significativa entre los cinco factores que definen la escala, con un 59,23\% de la varianza explicada, un índice de confiabilidad a través del Alfa de Cronbach $=0,83$ y con un buen ajuste (chi-cuadrada $=245,957 ; \mathrm{GI}=136 ; \mathrm{CMIN} / \mathrm{DF}=3,23 ; \mathrm{p}=0,000 ; \mathrm{CFI}=0,972 ; \mathrm{y}$ RMSE=0,047).

Conclusiones: Los hallazgos sugieren que la escala cuenta con propiedades psicométricas adecuadas para medir tecnodependencia en población mexicana. Con esta investigación se aporta una escala con la que se pretende estimular la investigación sobre esta variable en el país y que en un mediano plazo permitirá entender su etiología, estudiar la relación que mantiene con otros factores de riesgo psicosocial y diseñar programas de prevención e intervención con el fin de mejorar la relación de los usuarios, con las Tecnologías de la Información y la Comunicación, creando a su vez espacios de uso saludables.

Palabras clave: tecnodependencia; phubbing; generación muda; vida en redes sociales; uso compulsivo del celular.

\section{Abstract}

Introduction: The use of technology has positioned as an indispensable tool in the nowadays of the labor scholar, and even social spheres. With that, the involving with the technologies allows us to perform with higher productivity and efficiency. However, a disadaptative use can promote diverse conditions that influence in the health and social relations of the users.

Objective: It was designed a scale for the measure of technology dependence in people, integrated by five factors that were named Phubbing, Generation Mute, Use of technology while driving, Compulsive use of cellphone, and Life on social media.

Method: 1026 subjects participated, of which $63 \%$ were males. Quantitative, cross-sectional study, with non-experimental design and non-probability sampling, that had 1026 participants, of which $63 \%$ were men. The Technodependence Scale developed by the authors for the present study was used, made up of a total of 15 items divided into five factors (Cell phone use when driving, Life in social networks, Mute Generation, Compulsive cell phone use and Phubbing), in addition to sociodemographic variables, with a reliability index of 0,83 . 
Results: It was found statistically significant correlation between the five factors that define the scale, with a $59,23 \%$ of the explained variance, reliability index through Cronbach's alpha $=0,83$, and a good adjustment (chi-square= 245,957; GI=136; CMIN/DF= 3,23; $p=0,000 ; C F I=0,972 ;$ and RMSE=0,047).

Conclusions: The findings suggest that the scale has adequate psychometric properties to measure technodependence in the Mexican population. This research provides a scale with which it is intended to stimulate the research on this variable in the country and that in the medium term will allow understanding its etiology, studying its relationship with other psychosocial risk factors and designing prevention and intervention programs in order to improve the relationship of users, with Information and Communication Technologies, creating in turn healthy spaces for use.

Keywords: technology dependence; phubbing; generation mute; life on social media; compulsive cellphone use

\section{INTRODUCCIÓN}

En la sociedad contemporánea la utilización de la tecnología se ha vuelto fundamental para el trabajo, el estudio y las relaciones interpersonales de todo tipo. Con ello, el nivel de involucramiento que las tecnologías permean en nuestras actividades es mayúsculo; en diversos casos cuando el uso es eficiente se traduce en resultados de mayor productividad. No obstante, también está generando afecciones físicas y sociales en las personas cuando se da un uso desadaptativo.

Como es sabido, vivimos en un mundo conectado, donde la Internet forma parte de nuestra idiosincrasia. Las Tecnologías de la Información y de la Comunicación (TIC), están transformando de forma radical la sociedad, y no solo en lo referente a los aspectos económicos y profesionales sino también, en los aspectos culturales, de ocio, de acceso a la información, en la manera de construir el conocimiento, influye en cómo pensamos, en la forma de establecer relaciones sociales, la forma de comunicarnos, está modificando la forma de sentir y de comportarnos, es decir, está reconfigurando la forma de operar en el mundo (Baque, Solís, y Arcos, 2016; Pérez, 2011).

Estos comportamientos relacionados con el uso de las TIC conllevan a considerar afectaciones en el individuo, en su desempeño escolar y laboral, en sus relaciones personales, en su autoestima, emociones y demás dimensiones reportadas en estudios que señalan, cómo el uso de los teléfonos inteligentes se relaciona con afectaciones en salud mental incluyendo la depresión, el estrés crónico, inciden también en el ciclo sueño y vigilia (Villavicencio et al., 2020).

No obstante, se requieren más estudios en poblaciones específicas que permitan recabar evidencia científica sobre el fenómeno, para disponer de mayor información sobre la incidencia y características y con ello, establecer 
planes preventivos y correctivos de acción, que promuevan la ocurrencia de mayores resultados de índole positivo reduciendo con ello las afectaciones.

En ese sentido, en los estudios sobre tecnodependencia, tecnoestrés y tecnoadicción, se explica que los usuarios presentan consecuencias positivas y negativas, manifestando estrés hacia la tecnología y cómo el uso continuado de la misma puede tener como consecuencia trastornos incluyendo el rechazo (Maier et al., 2015; Tarafdar et al., 2011). También, el uso compulsivo del celular predice de manera negativa el tecnoestrés. Sin omitir el debate que existe en la literatura de lo que puede ser considerado una adicción conductual y cómo esta variable (tecnoadicción), incide en el estrés y el malestar en general del individuo, donde se muestra que a mayor adicción, mayor número de consecuencias negativas como ansiedad, tristeza, estrés y enojo, y a su vez, menos sentimientos de felicidad (Villavicencio et al., en prensa). Pareciera que se abre un panorama donde las personas requieren desarrollar ciertas habilidades para un uso eficiente de las TIC que resuelva sus necesidades y potencialice el resultado, y al mismo tiempo reducir las afectaciones en diversas esferas.

\subsection{Tecnodependencia}

Adentrándonos a las diferentes variables relacionadas con un uso desadaptativo, está la tecnodependencia o adicción a la tecnología, como la implicación excesiva del hombre con la máquina (Internet, computadora, teléfono móvil, videojuegos y televisión), específicamente a las TIC (Sánchez-Carbonell et al., 2008). Las circunstancias en las que vivimos nos han inmerso en la necesidad de requerir en exceso el uso de tecnologías para desarrollarnos, aprender, desempeñar actividades laborales, entretenimiento, y socializar, esto puede desencadenar un uso cada vez mayor e incluso caer en el abuso de las mismas, distanciándonos del propósito principal de las TIC. Refiriéndonos específicamente a una adicción comportamental que genera fenómenos de tolerancia y abstinencia similares a los producidos por abuso de sustancias (Toranzo, Marín y Morán, 2018).

Partiendo de la base que, el abuso se refiere al uso de la sustancia en situaciones riesgosas, asociadas a problemas legales, a problemas sociales o interpersonales; y también a su uso recurrente dejando de cumplir con responsabilidades (Contreras, Díaz, López, Caynas y García, 2012). Esta relación de abuso hacia las tecnologías se ve con mayor presencia en las generaciones más jóvenes (nativos digitales) debido a la sobre estimulación sensorial 
que reciben, mayor acceso a redes sociales, variedad de información que consultan, y la poca tolerancia a la ausencia de estos objetos tecnológicos (Toranzo, et al., 2018).

\subsection{Generación muda}

El uso excesivo de los teléfonos celulares predispone a un comportamiento no verbal, limitando la interacción cara a cara con la otra persona y el contacto con el entorno que nos rodea (Barrios, Bejar y Cauchos, 2017). Se causa un aislamiento del mundo real, las personas no pueden relacionarse si no es a través del celular; su forma de comunicación se vuelve únicamente escrita.

Derivado de esto, se acuñó el término Generation Mute en Inglaterra, para referirse a un fenómeno originado por el uso de los teléfonos inteligentes y las aplicaciones de mensajería instantánea, con mayor prevalencia en personas de 16 a 24 años de edad, quienes realizan sus conexiones fundamentalmente a través de texto escrito en redes sociales (Becerra, 2019; Marín-Díaz y Cabero-Almenara, 2019). No obstante, el hábito puede extenderse a otros grupos de edades. En México, 90,3 \% de jóvenes en esas edades, forman parte de la "generación muda" (Jiménez, 2019).

Un estudio realizado a 500 personas de entre 18 y 34 años, encontró que el $75 \%$ de los participantes preferían utilizar mensajes para comunicarse (Jiménez, 2019). También reveló que la causa del por qué se prefieren los mensajes sobre las llamadas es debido a que los mensajes se adaptan mejor a sus horarios y no toman mucho tiempo, contrario a una llamada telefónica. EI $63 \%$ de los encuestados mencionaron que los mensajes de texto interrumpen menos que una llamada y que los mensajes son la opción favorita para lograr comunicarse, debido a que consideran que son más personales y menos invasivos.

\subsection{Phubbing}

Los dispositivos tecnológicos invaden un espacio significativo en la vida de muchos individuos, es por ello que en el año 2013, en Australia, se introdujo por primera vez la palabra phubbing (Karadag, Tosuntas, Erzen, Duru, Bostan, Sahin, 2015), término formado a partir de las palabras inglesas phone (teléfono) y snubbing (desaire), es la práctica que comete una persona cuando ignora a sus interlocutores por revisar su dispositivo móvil, que deteriora las relaciones personales y exhibe el uso indiscriminado de tecnologías que han irrumpido en la vida cotidiana (Capilla, 2018). Tras diversos análisis se 
reflexiona sobre el efecto de las TIC para facilitar que la gente se comunique con las personas que están ausentes, mas no con las presentes; teniendo un efecto negativo donde se desconectan de los cercanos produciendo una incomodidad ante el hecho de ser ignorado por su acompañante, reflejando sentimientos de abandono, la persona se siente excluida, e incide también en la autoestima, el sentido de pertenencia, así como en el significado de la existencia y control (Clairá, 2013; Morfen, 2018).

El phubbing se ha venido acrecentando al paso de los años con el desarrollo de nuevas tecnologías, debido a que en variedad de situaciones se ignora a la persona que está enfrente, ya sea para contestar, ver alguna información, distracción o el simple hecho de una falta de socialización cara a cara. Se ha llegado al punto en el que las personas no conciben un mundo sin conexión, lo que les conlleva a una escasa habilidad en las relaciones interpersonales pero una excelente facilidad para trabajar con las TIC, de las cuales obtienen su satisfacción de entretenimiento, diversión, comunicación, información e incluso formación (Tavárez y Alvarado, 2018).

Dentro de las afectaciones de este fenómeno, se ha reportado en la literatura que en un matrimonio donde ocurre phubbing, las personas son más propensas a sufrir depresión y a tener una satisfacción marital menor. Un restaurante ve en promedio 36 casos de phubbing por sesión de cena, el $97 \%$ de las personas que han sufrido de esta variable reportan que su comida supo peor durante el incidente. La Ciudad de México se encuentra en el noveno lugar dentro de las ciudades con más phubbers, en ese listado ocupan los primeros tres lugares las ciudades de Nueva York, Los Ángeles y Londres, respectivamente (Morfen, 2018).

\subsection{Uso del celular al manejar}

Se estima que cada año 1,3 millones de defunciones y 50 millones de heridos son resultado de accidentes de tránsito. La distracción de los conductores es un factor a considerar, el uso del celular se ha convertido en el distractor número uno como causante de accidentes vehiculares, especialmente en jóvenes debido al constante uso y estimulación de los aparatos tecnológicos mientras se está al volante; tomarse selfies, llamar y mensajear por celular mientras se maneja, aumenta cuatro veces la probabilidad de sufrir accidentes (Organización Mundial de la Salud, 2011). 
La evidencia indica que el uso del celular cambia el nivel de atención cuando de manera simultánea conducen y realizan una llamada telefónica, aunque la mayoría de los usuarios reconoce que es peligroso hacer uso del teléfono mientras conducen, estos no dejan de hacerlo, lo que indica que existe un déficit en el control del uso del equipo telefónico (Agüero, Almeida, Espitia, Flores, y Espig, 2014). El incremento de accidentes automovilísticos por el uso del celular es provocado por ambas partes, los conductores que revisan el celular unos segundos mientras conducen y los peatones que dejan de percibir su alrededor al atender sus notificaciones.

Los resultados de la Encuesta del Mercado de Seguros Conectados indican que, en el país, el $49 \%$ de los encuestados reporta alta preocupación ante los conductores que utilizan su teléfono celular mientras manejan, y el $89 \%$ de los conductores reconoce haber visto al menos una vez al día a otros conductores haciendo uso de sus teléfonos celulares. El uso de teléfonos mientras se conduce se convirtió en la causa principal de muertes en accidentes de tráfico en México, el cual equivale a un $60 \%$, lo cual se coloca por encima de otras causas como lo son conducir a exceso de velocidad o en estado de ebriedad (Centro de Experimentación y Seguridad Vial México [CESVI], 2016).

Las distracciones causadas por usar el celular pueden ser visuales (desvían la vista del camino, por ejemplo, al ver la pantalla del celular para leer un mensaje); cognitivas (apartan la atención de la calle y del acto de manejar o caminar, como al pensar las respuestas en una conversación al teléfono); físicas (cuando se deja de usar una o ambas manos para conducir al responder una llamada o un mensaje en el celular); o auditivas (desvían la atención de los sonidos del tránsito como un claxon o ambulancia, durante las llamadas y al escuchar música) (Dirección General de Comunicación Social UNAM, s.f.). Cuando se hace uso del celular mientras se va manejando la atención cambia de prioridades, lo que provoca un desajuste en nuestras percepciones, lo que sumado a la velocidad en la que nos movemos puede provocar un accidente llegando a involucrar a terceros. De acuerdo con Ramos (2017) el uso del teléfono celular hace que el conductor desvíe la mirada de la carretera, quite las manos del volante y aparte su mente de la carretera y de la situación existente, lo que se le conoce como distracción cognitiva y es la que tiene mayores consecuencias en el comportamiento del conductor.

El uso excesivo de las nuevas tecnologías provoca ansiedad, adicción y desmemoria, asegura que en un rango de los 13 a los 22 años de edad, es 
el periodo en el que se desarrolla una mayor adicción al uso de los aparatos, en donde también pueden influir factores como una baja autoestima y la necesidad de aceptación, esto puede repercutir tanto en las relaciones sociales del individuo como en su desempeño, así como en el ámbito laboral (IMSS, 2018).

Las selfies, llamadas y textear se han convertido en el distractor número uno como causante de accidentes vehiculares, especialmente en jóvenes de 15 a 29 años, y su riesgo es equiparable al impacto que tiene manejar bajo los efectos del alcohol (Barragán, 2019). Los hábitos de los usuarios de internet en México incluyen un $67 \%$ de los internautas que se perciben conectados a internet las $24 \mathrm{hrs}$. Entre los principales usos que los mexicanos le brindan al internet, se encuentra en primer lugar el acceso a redes sociales con un $82 \%$, seguido de enviar/recibir mensajes instantáneos con el $78 \%$ y en tercer lugar, enviar/recibir mails con un $77 \%$. El $92 \%$ de los internautas mexicanos acceden a una conexión de internet a través de un teléfono inteligente (Asociación de Internet MX, 2019, p.15).

A fin de proporcionar un instrumento confiable para medir tecnodependencia, para explorar la ocurrencia de este fenómeno, y derivar posibles descriptores por edades, ámbitos de acción, que lleve a generar planes de intervención preventiva; la presente investigación tuvo por objetivo desarrollar una escala para población mexicana.

\section{MÉTODO}

\subsection{Participantes}

Participaron 1026 personas, siendo 647 hombres (63,1 \%) y 379 mujeres (36,9\%), con edades comprendidas entre los 15 a 92 años (media $=30,01$, $D E=13,82$ ), que tienen estudios de secundaria a posgrado. Se tomaron en cuenta factores como la edad, el género, el estado civil, el nivel escolar, si la persona cuenta con internet en casa, si tiene un plan de datos de navegación y en qué región de la República Mexicana radica (véase Tabla 1). Criterios de selección de la muestra: De inclusión: Personas de 15 años en adelante que deseen colaborar, personas que actualmente vivan en la República Mexicana, y con acceso a las tecnologías. De exclusión: Personas menores de 15 años de edad. Tipo de estudio: Exploratorio: El objetivo consistió en examinar un fenómeno poco estudiado en nuestro país. 
Tabla 1.

Características Sociodemográficas de los Participantes

\begin{tabular}{|c|c|c|c|}
\hline $\begin{array}{l}\text { VARIABLE } \\
\text { EDAD }\end{array}$ & $\begin{array}{c}\text { VARIABLES } \\
\text { RANGO: } 15 \text { A } 92 \text { AÑOS } \\
\text { MEDIA }=30,01, D E=13,82\end{array}$ & FRECUENCIA & PORCENTAJE \\
\hline \multirow{3}{*}{ Géneró } & Hombre & & $63,1 \%$ \\
\hline & Mujer & & $36,9 \%$ \\
\hline & Total & & $100 \%$ \\
\hline \multirow{6}{*}{ Estado Civil } & Soltero & & $67,7 \%$ \\
\hline & Casado & & $19,4 \%$ \\
\hline & Unión Libre & & $7,1 \%$ \\
\hline & Divorciado & & $5,0 \%$ \\
\hline & Viudo & & $0,8 \%$ \\
\hline & Total & & $100 \%$ \\
\hline \multirow{7}{*}{ Nivel Escolar } & Primaria & & $0,1 \%$ \\
\hline & Secundaria & & $3,1 \%$ \\
\hline & Bachillerato & & $28 \%$ \\
\hline & Técnico & & $5,1 \%$ \\
\hline & Licenciatura & & $51,1 \%$ \\
\hline & Posgrado & & $12,7 \%$ \\
\hline & Total & & $100 \%$ \\
\hline \multirow{3}{*}{ ¿Tienes Internet en casa? } & Sí & & $97,2 \%$ \\
\hline & No & & $2,8 \%$ \\
\hline & Total & & $100 \%$ \\
\hline \multirow{2}{*}{ ¿Tienes contratado algún plan de datos móviles? } & Sí & & $65,9 \%$ \\
\hline & No & & $34,1 \%$ \\
\hline \multirow{7}{*}{ Región república mexicana en donde vive. } & Total & & $100 \%$ \\
\hline & Noroeste & & $0,4 \%$ \\
\hline & Noreste & & $1,2 \%$ \\
\hline & Centro & & $93,2 \%$ \\
\hline & Occidente & & $2,9 \%$ \\
\hline & Sureste & & $2,3 \%$ \\
\hline & Total & & $100 \%$ \\
\hline
\end{tabular}

\subsection{Instrumentos}

Se crearon 33 reactivos divididos en nueve factores con una escala Likert de siete puntos que va nunca hasta siempre. Una vez realizados los análisis para la validación y comprobación de los resultados, se obtuvo la Escala de Tecnodependencia conformada por un total de 15 reactivos divididos en 5 factores, además de las variables sociodemográficas descritas arriba, con un índice de confiabilidad de 0,83 y una varianza explicada de 59,23 (véase Tabla 2). 
Tabla 2.

Escala de Tecnodependencia

\begin{tabular}{ccc}
\hline$\#$ & REACTIVO & FACTOR \\
\hline 14 & Suele hacer o recibir llamadas y mensajes cuando va manejando. & \\
15 & Aprovechas el semáforo en rojo para revisar el celular. & Uso del celular al conducir \\
13 & ¿Revisas el celular cuando vas manejando? & \\
9 & Siento la necesidad de compartir mis actividades en las redes sociales. & Vida en redes sociales \\
7 & Comparto aspectos de mi personalidad en las redes sociales. & Generación Muda \\
3 & Cuando asistes a un evento masivo, ¿̇sueles grabarlo? & Uso compulsivo del celular \\
1 & Me molesta recibir llamadas. & \\
4 & Cuando voy a realizar una llamada, pongo excusas para no hacerla. \\
8 & ¿Revisas el celular en cuanto te das cuenta que llega una notificación? & ¿Buscas estar siempre conectado a Internet? \\
10 & Cuando sales con alguien, cंsueles prestarle más atención al celular que a las personas con & Phubbing \\
11 & En reuniones, ¿̇sueles estar revisando por tiempo prolongado el celular? & \\
12 & Me gusta usar el celular mientras estoy comiendo aunque esté acompañado. &
\end{tabular}

\subsection{Procedimiento}

Se diseñó un formulario en Google con la escala, todas las preguntas eran de carácter obligatorio. Este formulario se distribuyó y aplicó a través de redes sociales tales como Facebook, Twitter, Instagram, Linkedin y WhatsApp, asimismo se compartió por medio de código QR en los planteles educativos visitados de nivel bachillerato y universidad, donde fue contestada por profesores, alumnos y personal administrativo. Una tercera forma de aplicación fue cara a cara a la gente en los sitios de común acceso.

\section{RESULTADOS}

El análisis factorial de componentes principales con rotación varimax de la escala de Tecnodependencia mostró cinco factores que explicaron el 59,23\% de la varianza. El primero se refiere al uso del celular al momento de conducir, entendido como realizar llamadas, envío de mensajes, uso de aplicaciones. El segundo es la vida en redes sociales, lo que involucra a las personas que comparten vídeos e imágenes la mayor parte del tiempo posible. El tercero es sobre la generación muda, mostrando características de los que prefieren enviar mensajes de texto y usar las redes sociales para comunicarse, en lugar de llamar por teléfono. El cuarto factor refiere al uso compulsivo del celular, cuando la persona responde ante cualquier estímulo irresistible que le presenta el celular creando un impulso y una conducta adictiva. Y el quinto mide la ocurrencia del Phubbing, para identificar las acciones que 
llevan a ignorar la presencia del otro para prestar atención al celular. Los cinco factores correlacionan positivamente. Se eliminaron dieciséis reactivos debido a que sus cargas factoriales fueron $<0,40$. Se calculó la confiabilidad del instrumento en esta primera etapa y obtuvo un $\alpha$ de Cronbach de 0,862

Al poner a prueba la estructura factorial resultante del análisis factorial exploratorio (varimax), mediante ecuaciones estructurales se eliminaron dos reactivos por los valores de ajuste. El coeficiente alpha de Cronbach fue de 0,83 para la escala total (véase Tabla 3).

Tabla 3.

Análisis, correlaciones, confiabilidad y validez de la Escala de Tecnodependencia

\begin{tabular}{|c|c|c|c|c|c|}
\hline \multirow[t]{2}{*}{ REACTIVOS } & \multicolumn{5}{|c|}{ SUBESCALAS } \\
\hline & $\begin{array}{l}\text { USO DEL } \\
\text { CELULAR } \\
\text { AL CON- } \\
\text { DUCIR }\end{array}$ & $\begin{array}{l}\text { VIDA EN } \\
\text { REDES } \\
\text { SOCIALES }\end{array}$ & $\begin{array}{l}\text { GENERACIÓN } \\
\text { MUDA }\end{array}$ & $\begin{array}{c}\text { USO } \\
\text { COMPUL- } \\
\text { SIVO DEL } \\
\text { CELULAR }\end{array}$ & PHUBBING \\
\hline $\begin{array}{l}\text { 15. Suele hacer o recibir llamadas y mensajes cuando vas } \\
\text { manejando. }\end{array}$ & 0,845 & & & & \\
\hline $\begin{array}{l}\text { 14. Aprovechas el semáforo en rojo para revisar el } \\
\text { celular. }\end{array}$ & 0,838 & & & & \\
\hline 13. ¿Revisas el celular cuando vas manejando? & 0,806 & & & & \\
\hline $\begin{array}{l}\text { 7. Siento la necesidad de compartir mis actividades en } \\
\text { las redes sociales. }\end{array}$ & & 0,975 & & & \\
\hline $\begin{array}{l}\text { 9. Comparto aspectos de mi personalidad en las redes } \\
\text { sociales. }\end{array}$ & & 0,672 & & & \\
\hline 5. Cuando asistes a un evento masivo, ¿sueles grabarlo? & & 0,521 & & & \\
\hline 3. Me molesta recibir llamadas. & & & 0,810 & & \\
\hline 1. Evito las llamadas que recibo. & & & 0,771 & & \\
\hline $\begin{array}{l}\text { 2. Cuando voy a realizar una llamada, pongo excusas } \\
\text { para no hacerla. }\end{array}$ & & & 0,688 & & \\
\hline $\begin{array}{l}\text { 4. ¿Revisas el celular en cuanto te das cuenta que llega } \\
\qquad \text { una notificación? }\end{array}$ & & & & 0,808 & \\
\hline 6. ¿Buscas estar siempre conectado a Internet? & & & & 0,769 & \\
\hline $\begin{array}{l}\text { 8. Siento la necesidad de estar revisando mis redes } \\
\text { sociales. }\end{array}$ & & & & 0,623 & \\
\hline $\begin{array}{l}\text { 10. Cuando sales con alguien, ¿̇sueles prestarle más } \\
\text { atención al celular que a las personas con las que estás? }\end{array}$ & & & & & 0,775 \\
\hline $\begin{array}{l}\text { 11. En reuniones, ¿̇sueles estar revisando por tiempo } \\
\text { prolongado el celular? }\end{array}$ & & & & & 0,713 \\
\hline $\begin{array}{l}\text { 12. Me gusta usar el celular mientras estoy comiendo, } \\
\text { aunque esté acompañado. }\end{array}$ & & & & & 0,576 \\
\hline
\end{tabular}




\begin{tabular}{|c|c|c|c|c|c|c|}
\hline Número de reactivos & 15 & 3 & 3 & 3 & 3 & 3 \\
\hline$\%$ de varianza explicada & 59,23 & 28,08 & 12,09 & 9,88 & 4,89 & 4,26 \\
\hline Alfa de Cronbach & 0,83 & 0,85 & 0,74 & 0,79 & 0,77 & 0,77 \\
\hline $\begin{array}{l}\text { Correlaciones inter factor } \\
\text { Vida en redes sociales } \\
\text { Generación Muda } \\
\text { Uso compulsivo del celular } \\
\text { Phubbing }\end{array}$ & & $\begin{array}{c}0,188^{*} \\
0,075 \\
0,211^{*} \\
0,295^{*}\end{array}$ & $\begin{array}{l}0,168^{*} \\
0,522^{*} \\
0,419^{*}\end{array}$ & $\begin{array}{l}0,187^{*} \\
0,285^{*}\end{array}$ & $0,479 *$ & \\
\hline $\begin{array}{l}\text { Media (media teórica = ) } \\
\text { Desviación estándar }\end{array}$ & & $\begin{array}{l}1,06 \\
1,50\end{array}$ & $\begin{array}{l}1,87 \\
1,43\end{array}$ & $\begin{array}{l}1,83 \\
1,48\end{array}$ & $\begin{array}{l}3,55 \\
1,50\end{array}$ & $\begin{array}{l}1,46 \\
1,24\end{array}$ \\
\hline KMO & 0,832 & & & & & \\
\hline Bartlett & 61785,371 & & & & & \\
\hline $\mathrm{Gl}$ & 105 & & & & & \\
\hline$P$ & 0,000 & & & & & \\
\hline
\end{tabular}

${ }^{*} p<0,000$

Como 0,83 $>$ 0,80 mínimo aceptable, el instrumento pasa la prueba de confiabilidad.

Los resultados obtenidos en KMO (puntaje 0,832) y en el Test de esfericidad de Bartlett, indicaron que era apropiado aplicar el análisis factorial (véase Tabla 3). Se puso a prueba la estructura factorial resultante del análisis factorial exploratorio (varimax) el instrumento final quedó compuesto por 15 reactivos que obtuvieron cargas factoriales significativas y lograron adaptarse al modelo diseñado en AMOS (ver Figura 1) cuyos índices de bondad de ajuste (ver Tabla 4) son aceptables (chi cuadrada $=245,957 ; \mathrm{Gl}=136 ; \mathrm{CMIN} / \mathrm{DF}=3,236 ; \mathrm{p}=0,000$; $\mathrm{CFI}=0,972$, y RMSEA $=0,047)$.

Tabla 4.

Estadísticos de bondad de ajuste de la escala de tecnodependencia

\begin{tabular}{cc}
\hline CMIN & 245,957 \\
DF & 76 \\
CMIN/DF & 3,236 \\
CFI & 0,972 \\
SRMR & 0,046 \\
RMSEA & 0,047 \\
PClose & 0,789 \\
CMIN & 245,957 \\
DF & 76 \\
CMIN/DF & 3,236 \\
\hline
\end{tabular}




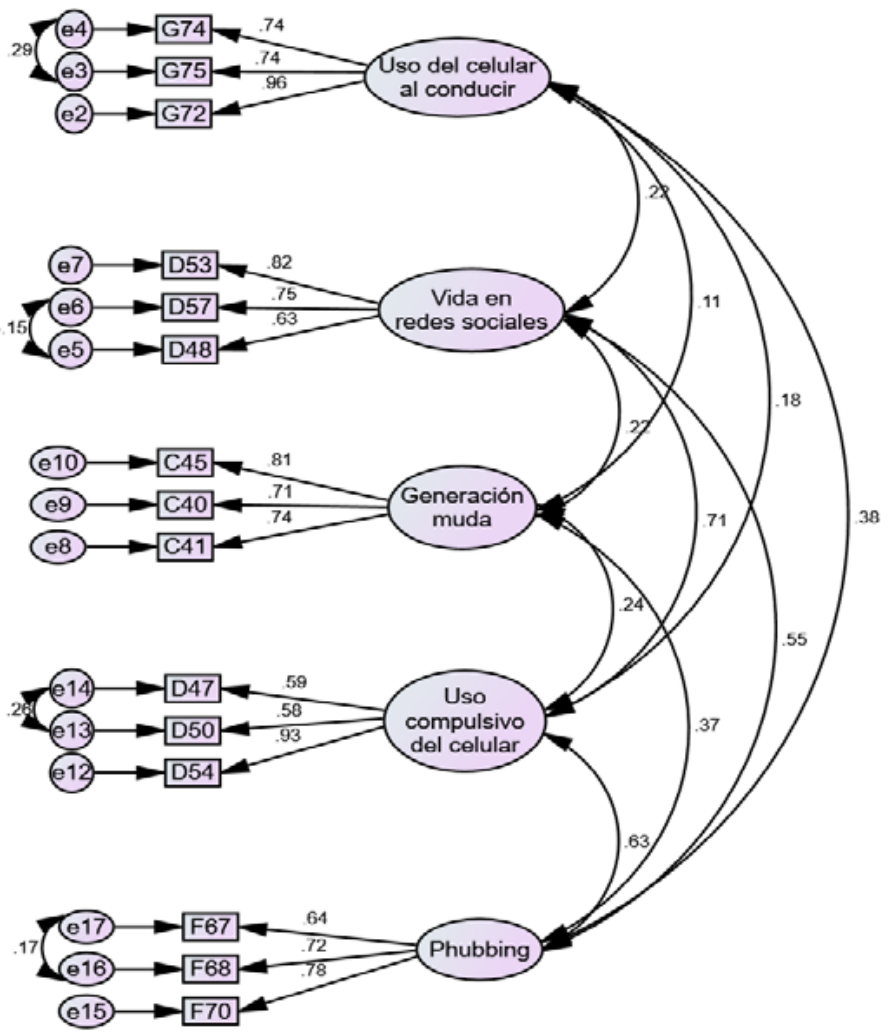

Figura 1. Modelo de estructura factorial de la escala de Tecnodependencia (elaboración propia)

\section{DISCUSIONES}

La implicación excesiva con la tecnología conocida como tecnodependencia conlleva a consecuencias nocivas para la salud (Sánchez-Carbonell et al., 2008); por ejemplo, el uso desmedido del celular puede ocasionar modificaciones del estado de ánimo, ansiedad, miedo a salir sin el dispositivo, tener que volver por él si se olvida y/o no ser capaz de hacer nada sin el móvil (Criado, 2005).

Asimismo, en el caso de los jóvenes con uso desadaptativo de las tecnologías de la información, desarrollan afectaciones en diversos contextos (Gracia Blanco et al., 2002), con elevados índices de depresión (Capilla \& Cubo, 2017), trastornos de ansiedad, introversión y trastornos de sueño. Llegando a priorizar la comunicación a través del celular, alterando sus contactos 
sociales migrando su interacción a una plataforma digital (Adès y Lejoyeux, 2001), afectando sus habilidades de interacción personal y comunicación verbal (Criado, 2005).

La tecnodependencia conlleva a una falta de control ante el uso de los dispositivos tecnológicos que van mermando la calidad de vida de los usuarios (Griffiths, 2017; Kardefelt-Winther, D. Heeren et al., 2017). Estas conductas pueden variar desde una ansiedad desmedida por no tener acceso al celular, reflejándose conductualmente en cambios de humor, reducción de relaciones sociales, afectación en la autoestima y hasta afectaciones físicas como cervicalgias, tendinitis, síndrome del túnel carpiano y alteraciones de la visión (Sánchez-Carbonell et al., 2008). Si contemplamos la actualidad, con la pandemia por Covid-19 se elevaron los niveles de trastornos asociados al uso de las tecnologías (Torres, 2021).

Sin embargo, no todo el uso de estas tecnologías lleva a consecuencias negativas, de igual forma en la pandemia sanitaria por Covid-19, ante las medidas tomadas para reducir la probabilidad de contagio, algunas personas establecieron conexiones a través de estos dispositivos tecnológicos, sin estar físicamente cerca, logrando con ello mantenerse en comunicación y no arriesgar su salud y la de sus seres queridos (Ahmed et al., 2021).

Cada una de las dimensiones que esta variable incluye, puede ser analizada en posteriores investigaciones para aportar más información de cómo se está manifestando en ciertas poblaciones, por ejemplo, cuando los jóvenes invierten demasiado tiempo en conocer la vida de los demás en redes sociales interiorizando un miedo mayor de volverse invisibles frente a su comunidad virtual si dejan de tener presencia en estos medios, o de mandar mensajes (Hall \& Baym, 2012). O también con el phubbing, cuando se presenta como la reducción de atención, interacción con la persona que está a tu lado y que tiende a disminuir considerablemente la interacción social (Roberts \& David, 2016), desarrollando por un lado la socialización a través de las redes sociales descuidando las físicas (Rozo Fonseca, 2016).

Se requiere de un trabajo por parte de los investigadores y usuarios para generar conciencia sobre la usanza que se le está dando a las tecnologías, con la finalidad de promover un equilibrio. Analizar los comportamientos que ya están presentándose y que lleva a afectaciones personales, familiares, laborales, de salud, entre otros; como por ejemplo el tener acceso a Internet ha provocado que no se respeten los horarios para cada actividad de la vida diaria, como el caso de un horario de trabajo, cuando se mandan mensajes a 
lo largo del día esperando respuesta, situación que hasta cierto punto se ha convertido en una exigencia laboral.

El desarrollar investigación sobre estos temas actuales, permite incluir herramientas para identificar las características de esta variable con diversos datos sociodemográficos y con ello desarrollar modelos de sensibilización y formación preventiva para la población, tanto niños, jóvenes y adultos, sobre la importancia de un uso eficiente de las TIC sin llegar a afectaciones en diversos ámbitos como por ejemplo la promoción de la seguridad vial, manejo de conflictos con la pareja derivados del phubbing, bajo desempeño escolar o laboral, entre otros.

Con los hallazgos de la presente investigación, se aporta una escala para la cultura mexicana, herramienta psicométrica que posibilita el estudio de la tecnodependencia y con ello obtener información objetiva con relación en los constructos ahí incluidos, así como conocer la situación que guarda la actual dependencia al uso de las TIC y por ende diseñar estrategias para promover acciones destinadas a diseñar programas de prevención y/o intervención con el fin de mejorar la relación entre los usuarios y las TIC, obteniendo el mayor provecho y reduciendo las afectaciones.

\section{CONCLUSIONES}

Los resultados obtenidos tras el proceso de diseño y validación, permitieron identificar que la escala de tecnodependencia aquí propuesta, es útil para medir los comportamientos que llevan a una implicación excesiva entre el hombre y las TIC.

El instrumento final quedó compuesto por quince reactivos distribuidos en 5 factores las cuales fueron denominadas como Uso del celular al conducir, Vida en redes sociales, Generación muda, Uso compulsivo del celular, y Phubbing; todos los reactivos obtuvieron cargas factoriales significativas y lograron adaptarse al modelo diseñado en AMOS cuyos índices de bondad de ajuste resultaron aceptables (chi cuadrada= 245,957; GI=136; CMIN/ $D F=3,236 ; p=0,000 ; C F I=0,972$, y RMSEA=0,047), que explican el 59,23\% de la varianza y coeficiente de confiabilidad Alpha de Cronbach de 0,83 , lo que muestra que es una escala que cumple con los parámetros estadísticos para usarse en población mexicana.

Se encontró también, que el uso del celular al conducir se encuentra relacionado positivamente con los otros cuatro factores, pero debido a que 
el manejar requiere requisitos tales como saber conducir, tener un vehículo y que el vehículo tenga conexión con el celular, además que realizar esta actividad involucra exponerse a un accidente o una multa/sanción no da pie a que exista una causalidad directa entre estas variables; es correcto señalar que existe cierto grado de relación entre las mismas.

La correlación entre los factores generación muda y vida en redes sociales es positiva débil $(0,168)$, debido a que el publicar en redes sociales no tiene como primer fin una comunicación directa, esta comunicación suele ser muy concreta y ocasionalmente generará una plática que se daría dentro de la misma red en mensajes personales. En cambio, el factor de Uso compulsivo del celular correlacionó alto con los factores de phubbing y vida en redes sociales $(0,479$ y 0,522 respectivamente), así como phubbing con vida en redes sociales $(0,419)$.

Conflicto de interés: Los autores declaran no tener conflicto de intereses.

Agradecimientos: Agradecemos la participación de la Facultad de Psicología de la UNAM.

Financiamiento: La presente investigación no contó con financiamiento.

\section{REFERENCIAS}

Adès, J. y Lejoyeux, M. (2001). Encore plus! Jeu, sexe, travail, argent. Odile Jacob

Agüero, D., Almeida, G., Espitia, M., Flores, A., y Espig, H. (2014). Uso del teléfono celular como distractor en la conducción de automóviles. Salus, 18(2), 27-34. https://www.redalyc.org/pdf/3759/375939026006.pdf

Ahmed, Z., Bin, S., Mohammed, S., y Islam, S. (2021). Smartphone overuse: A hidden crisis in COVID-19. Health Policy and Technology, 10-11. https://doi.org/10.1016/j. hlpt.2021.01.002

Asociación de Internet MX. (2019). 15 Estudio sobre los Hábitos de los Usuarios de Internet en México 2019. En Asociación de Internet MX. https://irp-cdn. multiscreensite.com/81280eda/files/uploaded/15\%2BEstudio\%2Bsobre\%2Blos\%2BHa_bitos\%2Bde\%2Blos\%2BUsuarios\%2Bde\%2BInternet\%2Ben\%2BMe_xico\%2B2019\%2Bversio_n\%2Bpu_blica.pdf

Baque, O., Solís, H., \& Arcos, Á. (2016). La Tecnodependencia: libertad o sujeción. Revista Contribuciones a las Ciencias Sociales. https://www.eumed.net/rev/ cccss/2016/03/tecnodependencia.html

Barragán. E. (2019). Jóvenes mexicanos pertenecen a "generación muda". En Siete24. https://siete24.mx/tendencias/jovenes-mexicanos-pertenecen-a-generacion-muda/

Barrios, D. A., Bejar, V., \& Cauchos, V. (2017). Uso excesivo de Smartphones/teléfonos celulares: Phubbing y Nomofobia. Revista chilena de neuro-psiquiatría, 55(3), 205-206. http://dx.doi.org/10.4067/s0717-92272017000300205 
Becerra, J. (2019). Cio México. http://cio.com.mx/un-90-de-jovenes-forman-la-generacion-muda-en-mexico/

Capilla, R. (2018). Phubbing, ¿̇es más importante tu smartphone?. En Ciencia MX. http://www.cienciamx.com/index.php/tecnologia/tic/20437-phubbing-enmexico

Capilla, G. E., \& Cubo, D. S. (2017). Phubing. Conectados a la red y desconectados de la realidad. Un análisis en relación al bienestar psicológico. Pixel Bit, 50, 173-185. http://dx.doi.org/10.12795/pixelbit.2017.i50.12

Carbonell-Sánchez, X. (2014). Adicciones tecnológicas: ¿Qué son y cómo tratarlas? (Editorial).

Centro de Experimentación y Seguridad Vial México [CESVI]. (2016). Niños y jóvenes continúan mortalmente expuestos en accidentes viales. El Universal. https:// www.eluniversal.com.mx/articulo/autopistas/2016/04/28/cesvi-ninos-y-jovenes-continuan-mortalmente-expuestos-en-accidentes

Clairá, A. (2013). ¿Conectados o comunicados? Revista CN, 541. Recuperado de http://www.ciudadnueva.org.ar/revista/ 541/sociedad/conectados-o-comunicados

Contreras, E., Díaz, M., López, R., Caynas, S., \& García, P. (2012). El cerebro adicto. Rev med UV. Volumen especial 2012. 43-50 http://www.biblioteca.cij.gob.mx/ Archivos/Materiales_de_consulta/Drogas_de_Abuso/Articulos/Neurobiologia2. pdf

Criado, M. A. (2005). Adictos al móvil. http://www.el-mundo.es/\%0Aariadna/2005/218/1106327395.html

Dirección General de Comunicación Social UNAM. (s.f.). Uso de celular, riesgo para la seguridad de los peatones. En Fundación UNAM. https://www.fundacionunam. org.mx/unam-al-dia/uso-de-celular-riesgo-para-la-seguridad-de-los-peatones/

Echeburúa, E., \& Corral, P. (1994). Adicciones psicológicas: Más allá de la metáfora. Clínica y Salud, 5, 251-258. https://journals.copmadrid.org/clysa/art/07e1cd7dca89a1678042477183b7ac3f

Gialamas, V., Nikolopouiou, K., y Kutromanos, G. (2013). Student teachers' perceptions about the impact of internet usage on their learning and jobs. Computers \& Education, 62, 1-7. https://doi.org/10.1016 / j.compedu.2012.10.012

Gracia Blanco, M. de, \& Vigo Anglada, M., Fernández Pérez, M. J. \& Marcó Arbonès, M. (2002). Problemas conductuales relacionados con el uso de Internet: Un estudio exploratorio. Anales de Psicología, 18(2), 273-292. [Fecha de Consulta 15 de septiembre de 2021]. ISSN: 0212-9728. https://www.redalyc.org/articulo. oa? id $=16718206$

Griffiths, M. D. (2017). Behavioural addiction and substance addiction should be defined by their similarities not their dissimilarities. En Addiction (Vol. 112, Issue 10, pp.1718-1720). https://doi.org/10.1111/add.13828

Hall, J. A., \& Baym, N. K. (2012). Calling and texting (too much): Mobile maintenance expectations, (over)dependence, entrapment, and friendship satisfaction. New Media and Society, 14(2), 316-331. https://doi.org/10.1177/1461444811415047

Hernández, F. (2019). Los riesgos de las tecnologías de la información y la comunicación. CONAMED, 184-199. https://www.medigraphic.com/pdfs/conamed/ con-2019/con194d.pdf

Hernández, R., Fernández, C. \& Baptista, P. (2014). Selección de la muestra. En Metodología de la Investigación (6ª ed., pp.170-191). México: McGraw-Hill. 
IMSS (2018). La Adicción a las Nuevas Tecnologías Provoca Ansiedad y Desmemoria, Advierten Especialistas del IMSS. http://www.imss.gob.mx/prensa/ archivo/201808/202

Jiménez, P. (11 de agosto de 2019). ¿Por qué los millennials prefieren los mensajes a las Ilamadas? El Universal. https://www.eluniversal.com.mx/techbit/por-que-los-miIlennials-prefieren-los-mensajes-las-llamadas

Karadağ, E., Tosuntaş, ŞB., Erzen, E., Duru, P., Bostan, N. \& Şahin, BM. (2015). Determinants of phubbing, which is the sum of many virtual addictions: a structural equation model. J Behav Addict, 4(2): 60-74. https://doi.org10.1556 / 2006.4.2015.005

Kardefelt-Winther, D. Heeren, A., Schimmenti, A., van Rooij, A., Maurage, P., Carras, M., Edman, J., Blaszczynski, A., Khazaal, Y., \& Billieux, J. (2017). How can we conceptualize behavioural addiction without pathologizing common behaviours? Addiction.

Maier, C., Laumer, S., Eckhardt, A., \& Weitzel, T. (2015). Giving too much social support: Social overload on social networking sites. European Journal of Information Systems, 24(5), 447-464. https://doi.org/10.1057/ejis.2014.3

Marín, V., y Cabero, J. (2019). Las redes sociales en educación: desde la innovación a la investigación educativa? RIED. Revista Iberoamericana de Educación a Distancia, 22(2), pp.25-33. https://doi.org/10.5944/ried.22.2.24248

Morfen, M. (2018). ¿Qué es el 'phubbing' y por qué está arruinando tus relaciones? En Excelsior. https://www.excelsior.com.mx/trending/que-es-el-phubbing-y-porque-esta-arruinando-tus-relaciones/1230745

Organización Mundial de la Salud. (2011). Uso del celular al volante: un problema creciente de distracción del conductor. En Organización Mundial de la Salud. https://apps.who.int/iris/bitstream/handle/10665/85873/9789243500898_spa. pdf; jsessionid=87CB2AF3A6486A2DE3DEF0DE82931476? sequence=1

Pérez, A. (2011). Escuela 2.0 educación para el mundo digital. Revista de estudios de juventud, 92, 63-86. https://dialnet.unirioja.es/servlet/articulo?codigo=5047335

Ramos, I. (2017). El grado de conciencia de los jóvenes entre 18 y 28 años de edad del uso del celular al conducir. Honduras. UNITEC.

Roberts, J. A., \& David, M. E. (2016). My life has become a major distraction from my cell phone: Partner phubbing and relationship satisfaction among romantic partners. Computers in Human Behavior, 54, 134-141. https://doi.org/10.1016/j. chb.2015.07.058

Rozo Fonseca, M. (2016). La infoxicación, ¿existe o no en las grandes multinacionales? Tecnología, Investigación y Academia, 4(2), 6-11. http://orcid.org/00000002-7966-7611

Salazar, C. (2019). El Tecnoestrés y su efecto sobre la productividad individual y sobre el estrés de rol en trabajadores chilenos: un estudio psicométrico y predictivo (Tesis doctoral). Universidad Oberta de Catalunya.https://www.tdx.cat/bitstream/ handle/10803/668131/Tesis\%2BDoctoral\%2BEl\%2BTecnoestr_s\%2By\%2Bsu\%2Befecto\%2Bsobre\%2Bla\%2Bproductividad\%2Bindividual\%2By\%2Bsobre\%2Bel\%2Bestr s\%2Bde\%2Brol\%2Ben\%2Btrabajadores\%2Bchilenos\%2B-un\%2Bestudio\%2Bpsicom_trico\%2By\%2Bpredictivo-.pdf?sequence=1\&isAllowed=y

Sánchez-Carbonell, X., Beranuy, M., Castellana, M., Chamarro, A., \& Oberst, U. (2008). La adicción a Internet y al móvil: ¿̇moda o trastorno? Adicciones, 20(2), 149. https://doi.org/10.20882/adicciones.279.

Soto, M. (2016). Ojo con la dependencia tecnológica. El Tiempo. https://www. eltiempo.com/tecnosfera/novedades-tecnologia/dependencia-tecnologica-42096 
Tarafdar, M., Tu, Q., Ragu-Nathan, T. S., \& Ragu-Nathan, B. S. (2011). Crossing to the dark side: Examining creators, outcomes, and inhibitors of technostress. Communications of the ACM, 54(9), 113-120. https://doi.org/10.1145/1995376.1995403

Tavárez E. \& Alvarado, K. (2018). Análisis del perfil actitudinal frente al futuro académico de la generación $z$, para detectar oportunidades de mejora en el modelo de Enseñanza-Aprendizaje en la carrera de marketing y negociación Comercial de la Universidad de Guayaquil, año 2017. Bachelor's thesis, Universidad de Guayaquil Facultad de Ciencias Administrativas. http://repositorio. ug.edu.ec/bitstream/redug/39707/1/TESIS\%20MKT\%20EDUCACIONAL-\%20 TAVAREZ-ALVARADO.pdf

Toranzo, E., Marín, A., \& Morán, G. (2018). Relación entre las Bases Neurobiológicas de las Adicciones a Sustancias y las Tecnoadicciones. Anuario de Investigaciones de la Facultad de Psicología, 3(3), 560-568. https://revistas. psi.unc.edu.ar/index. php/aifp/article/view/20761

Torres, C. C. (2021). Adaptation and Validation of Technostress Creators and Technostress Inhibitors Inventories in a Spanish-Speaking Latin American Country. Technology in Society, 66(June), 101660. https://doi.org/10.1016/j. techsoc.2021.101660

Villavicencio-Ayub, E., \& Cazares, M. (2021). Adaptación y validación de la escala de tecnoadicción del cuestionario red- tecnoestrés, en una población laboral mexicana. Psicología Iberoamericana, 29(1), 1-19. https://www.redalyc.org/ articulo.oa?id=133967526002

Villavicencio-Ayub, E., Ibarra Aguilar, D. \& Calleja, N. (2020). Tecnoestrés en población mexicana y su relación con variables sociodemográficas y laborales. Psicogente, 23(44), 1-27. https://doi.org/10.17081/psico.23.44.3473

Villavicencio-Ayub, García, Quiroz, Callejo, Lagos (en prensa). Tecnodependencia, tecnoestrés y tecnoadicción: un análisis de sus consecuencias físicas y emocionales en población mexicana y colombiana. Cuadernos de Administración.

Zheng, X., \& Lee, M. K. O. (2016). Excessive use of mobile social networking sites: Negative consequences on individuals. Computers in Human Behavior, 65, 65-76. https://doi.org/10.1016/j.chb.2016.08.011

Esta obra está bajo: Creative commons attribution 4.0 international license. El beneficiario de la licencia tiene el derecho de copiar, distribuir, exhibir y representar la obra y hacer obras derivadas siempre y cuando reconozca y cite la obra de la forma especificada por el autor o el licenciante.

\section{(cc) BY}

